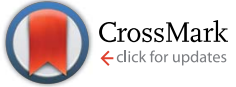

Cite this: Soft Matter, 2014, 10, 6514

Received 14th February 2014

Accepted 30th May 2014

DOI: $10.1039 / c 4 s m 00359 d$

www.rsc.org/softmatter

\section{Shear-driven aggregation of SU-8 microrods in suspension $\dagger$}

\author{
Pramukta Kumar, ${ }^{a}$ David Gold, ${ }^{a}$ Daniel L. Blair, ${ }^{a}$ Aparna Baskaran ${ }^{b}$ \\ and Jeffrey S. Urbach*a
}

A non-Brownian suspension of micron scale rods exhibits reversible shear-driven formation of disordered aggregates resulting in dramatic viscosity enhancement at low shear rates. Aggregate formation is imaged using a combined rheometer and fluorescence microscope. The size and structure of these aggregates are found to be a function of shear rate and concentration, with larger aggregates present at lower shear rates and higher concentrations. Quantitative measurements of the early-stage aggregation process are modeled by collision driven growth of porous structures which suggest that the aggregate density increases with shear rate. This result is combined with a Krieger-Dougherty type constitutive relationship and steady-state viscosity measurements to estimate the intrinsic viscosity of complex structures developed under shear. These results represent a direct, quantitative, experimental demonstration of the association between aggregation and viscosity enhancement for a rod suspension, and demonstrate a way of inferring microscopic geometric properties of a dynamic system through the combination of quantitative imaging and rheology.

\section{Introduction}

Suspensions of non-Brownian rod-like particles are found in a variety of engineered contexts, such as the processing of fiber reinforced composites and paper products. ${ }^{\mathbf{1 , 2}}$ These systems often exhibit dramatic rheological properties such as shearthinning ${ }^{3}$ and shear-thickening, ${ }^{4}$ governed by the particle size and shape, properties of the suspending fluid, interactions between the particles and the overall flow history. In particular, the formation of secondary structures such as aggregates and bundles is often responsible for very large rheological and electrical effects, ${ }^{5}$ as well as some of the most difficult material processing challenges. ${ }^{6}$

The dynamical processes involved in aggregate nucleation and growth are complex and share many features with shearinduced aggregation of spherical and small aspect ratio particles. ${ }^{7}$ For spherical particles, several studies have compared models of aggregate growth with direct measurements of aggregate size and structure. ${ }^{8-10}$ Though some simulation work has been done, ${ }^{11,12}$ the literature for fibers or rod-like particles is much less complete, and substantial open questions remain about pathways to aggregation and the structure of the aggregated phase.

\footnotetext{
${ }^{a}$ Dept. of Physics and Institute for Soft Matter Synthesis and Metrology, Georgetown University, Washington,DC, USA.E-mail: psk7@georgetown.edu ${ }^{b}$ Martin A. Fisher School of Physics, Brandeis University, Waltham, MA, USA $\dagger$ Electronic supplementary information (ESI) available: Three movies. See DOI: 10.1039/c4sm00359d
}

In this paper, we apply quantitative image analysis coupled with bulk rheological measurements to a model suspension of fluorescent polymer microrods that is reversibly driven between dispersed and aggregated states. We examine the micro- and meso-structural aggregation process and its relationship to the observed suspension viscosity by analyzing time-series images of the sheared suspension via confocal and wide-field fluorescence microscopy. We then use a simple early-stage aggregation model to infer the density of the aggregated phase. Finally, we use the density of the aggregated phase along with the KriegerDougherty constitutive relationship to describe the steady-state viscosity of the aggregated suspension. These results represent a direct, quantitative, experimental demonstration of the association between the aggregate size and viscosity enhancement in a rod suspension, and show the promise of this approach for advancing a first-principles understanding of the behavior of high aspect ratio rod suspensions.

\section{Experimental materials and methods}

\subsection{Microrod suspension}

Polymer microrods made of SU-8 photoresist (SU-8 25 by Microchem Corp.) were fabricated using the method of Alargova et al. with the added step of incorporating fluorescein salt into the photoresist prior to synthesis. ${ }^{13} 150 \mathrm{~mL}$ of $50 \%$ by volume aqueous Glycerol mixture was sheared at high speed in a stand mixer. While the solution was being mixed, $0.2 \mathrm{~mL}$ of the fluorescein and SU-8 mixture was injected under the impeller 


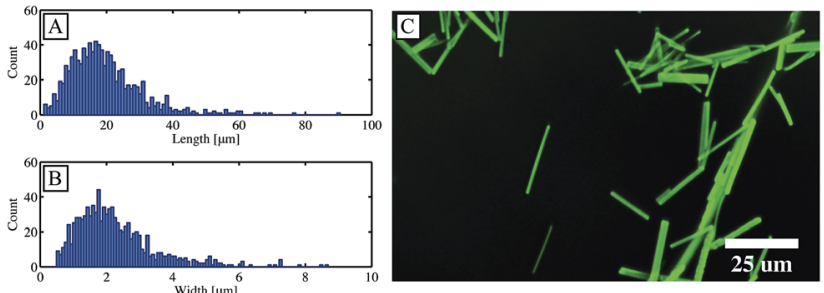

Fig. 1 Representative (A) length, and (B) width distributions of polydisperse SU- 8 microrods, shown in (C). The mean length of the rods is $19.8 \mu \mathrm{m}$ with a standard deviation of $11.0 \mu \mathrm{m}$, and the mean diameter is $2.27 \mu \mathrm{m}$, with a standard deviation of $1.18 \mu \mathrm{m}$

and subject to shear forces for 15 minutes. The resulting rod suspension was cross-linked in a wide bottom dish placed under $366 \mathrm{~nm}$ UV light for 45 minutes, then centrifuged for 30 minutes at $3 \times 10^{3} \mathrm{~g}$ in order to remove the largest particles.

The suspension was then filtered through $60 \mu \mathrm{m}$ and $43 \mu \mathrm{m}$ nylon filters (Membrane Solutions, LLC), followed by a $30 \mu \mathrm{m}$ etched filter (Sterlitech Inc). The remaining rods were captured by filtering through a $1 \mu \mathrm{m}$ track-etched filter (Millipore Inc). The rods trapped by this filter were resuspended into a fresh aqueous glycerol solution of matching density $\left(1.2 \mathrm{~g} \mathrm{~mL}^{-1}\right)$, and stored in darkness at $4{ }^{\circ} \mathrm{C}$.

The synthesis and filtration process resulted in a polydisperse suspension of microrods having an average length of $19.8 \mu \mathrm{m}$ and an average diameter of $2.27 \mu \mathrm{m}$ determined by directly imaging aqueous rod suspensions that have been made to settle on a cover slip (Fig. 1). Standard deviations in length and diameter were found to be $11.0 \mu \mathrm{m}$ and $1.18 \mu \mathrm{m}$, respectively. Two concentrations were used, $\phi=0.07$ and $\phi=0.04$, determined by thresholding confocal volumes of dispersed samples, created by controlled dilution.

\subsection{Rheology}

Steady-state and transient viscosity measurements were performed using a rheometer (MCR-301 by Anton Paar GmbH) fitted with a $25 \mathrm{~mm}$ parallel plate tool set $200 \mu \mathrm{m}$ above a stainless steel base plate. Temperature was held at $25{ }^{\circ} \mathrm{C}$ using Peltier-based base and hood temperature control devices (P-PTD 200 by Anton Paar GmbH). Nitrogen was circulated through the sample chamber in order to mitigate the hygroscopic tendency of the glycerol used in the suspension solvent.

Prior to each measurement series, the system was initialized by application of high shear $\left(1000 \mathrm{~s}^{-1}\right)$ for 10 minutes in order to fully disperse the suspended microrods. Steady-state viscosity measurements were made by letting the rheometer determine the integration time necessary to achieve a stable result. Transient viscosity measurements were taken at 1 second intervals.

\subsection{Rheo-microscopy}

The rheometer described above was coupled to an inverted microscope (DMI4000b by Leica Microsystems $\mathrm{GmbH}$ ) as shown in Fig. 2 in order to collect wide-field fluorescence images. To enable optical access, a measuring cup with a glass
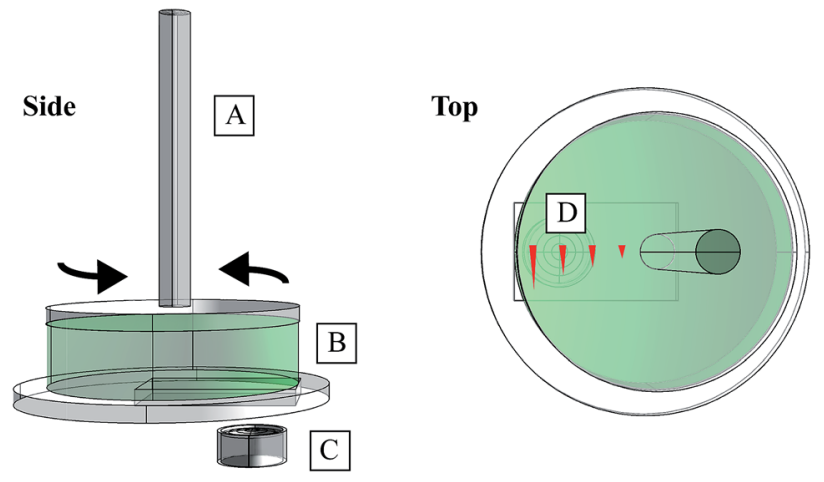

Fig. 2 Using a microscope objective (C) and associated optics, we gather fluorescence images of the sample fluid (B) as it is being sheared by using a rotating parallel plate rheometer tool (A). The rheometer tool sets up a shear gradient that increases towards the edge of the tool (D).

cover slip mounted inside was used in place of the standard stainless steel base plate. Fluorescence images were captured using an sCMOS camera (pco.edge by PCO AG) through a $1.6 \times$ objective. The sample temperature was controlled at $25{ }^{\circ} \mathrm{C}$ by using a bath circulator (Isotemp 3006 by Thermo Fisher Scientific Inc.) to pump water through a copper radiator surrounding the measuring cup. The sample was isolated from atmospheric moisture by immersing the outside edge of the measuring tool in a light mineral oil (Fisher Scientific Inc.).

The system was initialized just as for the pure rheological experiments. Images were collected at 1 second intervals using a shutter to synchronize illumination with the camera exposure in order to minimize photobleaching. Despite this precaution, photobleaching did occur over the duration of the experiment and was corrected in post-processing.

Confocal rheology ${ }^{\mathbf{1 4}}$ was also employed, using an identical rheometer (MCR-301 by Anton Paar GmbH) coupled to a confocal microscope (SP5 by Leica Microsystems $\mathrm{GmbH}$ ) with a $63 \times$ water immersion objective, illuminated by a $488 \mathrm{~nm}$ argon laser, and captured using a PMT detector. Images collected from this instrument show, in detail, the three-dimensional structures that form in the microrod suspensions (Fig. 7).

\subsection{Aggregate length scale}

Images of the suspension were median filtered using a $3 \times 3$ window to eliminate single pixel noise. Due to the shear induced flow field as well as the high time resolution of the images, intra-frame as well as inter-frame photobleaching effects were present in the data. These were removed by normalizing the intensity at each pixel by the local average in a surrounding 201 pixel $(822 \mu \mathrm{m})$ square window.

The tool's center coordinate was calculated by computing PIV streamlines and fitting them to circles. This allowed the calculation of one dimensional constant shear rate arcs along which to measure intensity profiles. Analysis was confined to arcs located at radii between $7.2 \mathrm{~mm}$ and $8.8 \mathrm{~mm}$ so as to stay away from edge and central effects. Arcs were spaced radially by 20 pixels $(82 \mu \mathrm{m})$. 
Aggregate length scales were computed by calculating onedimensional intensity autocorrelation functions along the previously calculated constant shear rate arcs. Intensity profiles were re-gridded to 1 pixel $(4.09 \mu \mathrm{m})$ spacings along the specified arcs. Autocorrelations were computed as

$$
R(s)=\frac{\left\langle\left(I_{x}-\bar{I}\right)\left(I_{x-s}-\bar{I}\right)\right\rangle}{\operatorname{var}(I)}
$$

where $I_{x}$ is the pixel intensity at a point $x, \bar{I}$ the mean pixel intensity, and $\operatorname{var}(I)$ the variance of the pixel intensity. Brackets represent an ensemble average over all samples of lag $s$. The length scale was then taken as the spatial lag associated with the autocorrelation function decaying to $1 / e(\min (s) \mid R(s)=1 / e)$.

\section{Results}

The steady-state suspension viscosity revealed a characteristic shear-thinning behavior (Fig. 3A) seen in many different systems including suspensions of latex spheres, ${ }^{15}$ cellulose whiskers, ${ }^{\mathbf{1 6}}$ carbon black, and carbon nanotubes. ${ }^{7}$ Both increasing and decreasing shear rate ramps exhibited identical behavior with no observed hysteresis. As the shear rate was increased, the overall suspension viscosity approached the solvent viscosity. An initially dispersed system exhibited a
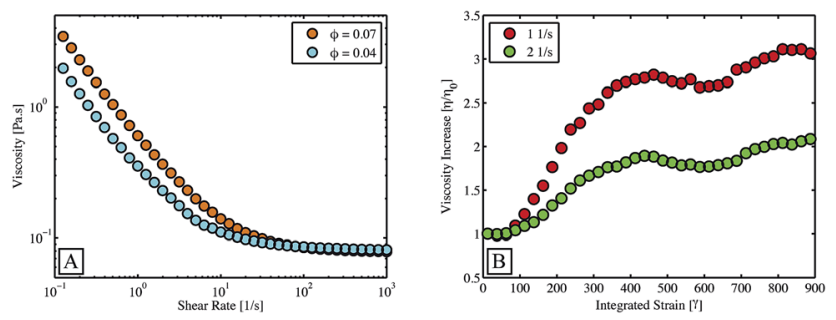

Fig. 3 (A) Representative steady-state viscosity curves for polydisperse SU- 8 microrod suspensions at two different concentrations measured with a $25 \mathrm{~mm}$ parallel plate rheometer tool with a $0.2 \mathrm{~mm}$ gap. The system may be reversibly driven between aggregated to dispersed states with no hysteresis. (B) Representative transient viscosity measurements as aggregates form starting from a dispersed state. Measurements were made under conditions identical to the steady-state curves. transient viscosity increase that eventually reached a steadystate plateau (Fig. 3B).

Direct visualization of the SU-8 microrod suspension under shear via wide-field fluorescence microscopy showed coarsening and subsequent formation of aggregates beginning at the edge of the tool where both shear rate and integrated strain are largest (Fig. 4 and ESI Movie $\dagger$ ). Aggregates formed were reminiscent of those seen in systems of carbon nanotubes ${ }^{17,18}$ and cellulose systems. ${ }^{19}$ The region containing aggregates subsequently expanded toward the center until it effectively spans the field of view. Aggregates formed under shear were observed to be completely broken up by high shear, resulting in a system that may be reversibly evolved from a dispersed to aggregated state and back again.

The aggregate size, as measured via intensity autocorrelation (eqn (1) and Fig. 5A), was found to increase with applied strain (Fig. 5C and D) until a steady-state aggregate size was reached at after a few hundred integrated strain units. Differences in steady-state aggregate size were observed between different shear rates (Fig. 5B). The curves representing aggregate size versus strain closely followed the measured viscosity increase (Fig. 3B), both in curve shape and in the location of the point of inflection.

We have analyzed the early-stage aggregation with a simple kinetic model, similar to those used for suspensions of low aspect ratio particles. ${ }^{\mathbf{9} 20}$ Assuming non-fractal growth of porous aggregates, the relationship between the aggregate radius and the number of particles in an aggregate can be written as

$$
n=\alpha \frac{V_{\mathrm{agg}}}{V_{\text {rod }}}=\frac{\alpha(4 / 3) \pi r^{3}}{V_{\text {rod }}} \rightarrow r=\left(\frac{n V_{\text {rod }}}{\alpha(4 / 3) \pi}\right)^{1 / 3}
$$

where $n$ is the number of rods in the aggregate, $r$ is the radius of the aggregate, and $\alpha$ represents the volume fraction of particles in an aggregate. $V_{\text {rod }}$ and $V_{\text {agg }}$ represent the volume of a rod and the total volume of the aggregate, respectively. We assume that, in the early-stage, low shear rate limit, all particles encountered by the aggregate as it sweeps through a dispersed field (of density $\rho$ ) will join the aggregate. The time evolution of the size of the aggregate will thus follow

$$
\frac{\mathrm{d} n}{\mathrm{~d} t}=\dot{\gamma} r \rho r^{2}
$$
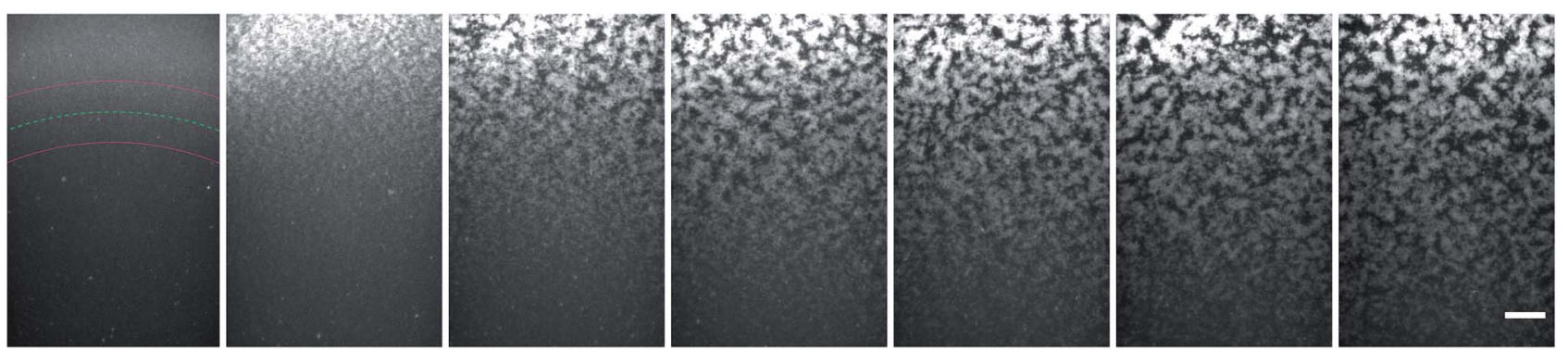

Fig. 4 Aggregation of an $\phi=0.07 \mathrm{SU}-8$ microrod suspension subjected to steady shear ( $1.5 \mathrm{~s}^{-1}$ edge shear rate), starting from a dispersed state. From left to right, the images were taken at $0,50,100,150,200,250$, and 300 seconds from the start of shearing. The region considered for analysis is highlighted in the first image of the series. The flow direction is tangential to a circle whose center is located below the displayed images, slightly out of frame. The scale bar represents $1 \mathrm{~mm}$. The full time evolution can be seen in the ESI movie. $\dagger$ 

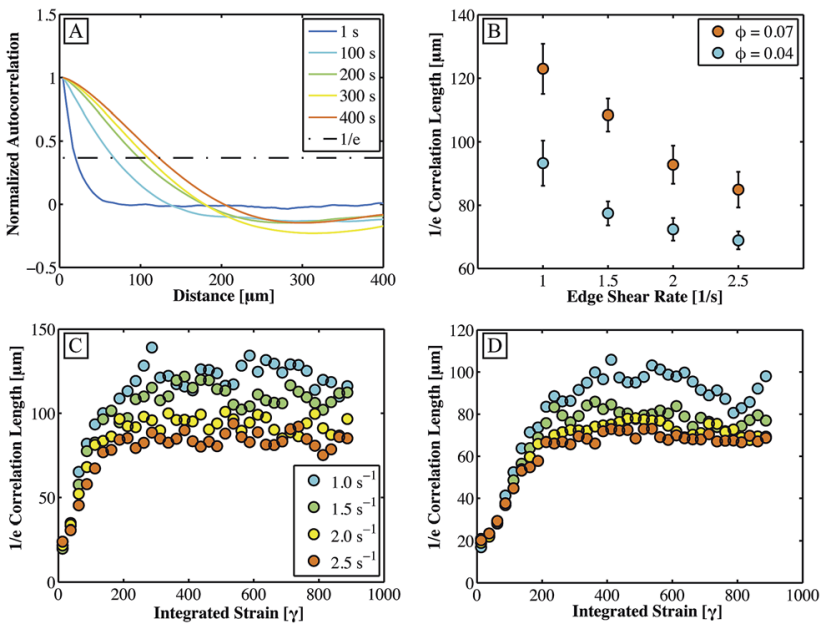

Fig. 5 (A) Intensity autocorrelations (eqn (1)) at different times after the start of shearing are shown for $\phi=0.07$ concentration suspensions, with an applied shear rate of $1 \mathrm{~s}^{-1}$. The aggregate length scale was defined to be the length at which the normalized autocorrelation function crosses 1/e. (B) The steady-state aggregate length scale decreased with increasing shear rate in a manner reminiscent of steady-state viscosity measurements (Fig. 3). (C and D) Aggregate length scale increased as shear-induced structures form in (C) $\phi=$ 0.07 and (D) $\phi=0.04 \mathrm{SU}-8$ microrod solutions. Higher concentrations appeared to aggregate faster than lower concentrations consistent with shear-driven collision based processes.

where $\dot{\gamma} r$ represents the relative velocity of the suspension and the aggregate, and $r^{2}$ represents the aggregate cross-sectional area. Due to the complexity of the aggregate shape, there is an unknown geometric factor of order unity ( $4 / 3$ for perfectly spherical aggregates) that would change the precise values for the density, but not the observed shear rate dependence.

Subsequent substitution and integration lead to the predicted relationship between the aggregate size and integrated strain,

$$
r(\gamma)=A \exp \left(\frac{V_{\mathrm{rod}} \rho \gamma}{4 \pi \alpha}\right)
$$

This relationship was fit to the experimentally measured early-stage aggregate growth, using $\alpha$ and $A$ as the free parameters. The fit range was chosen independently for each curve starting from the strain where the aggregate length first started to increase, and ending before nearing the steady-state plateau. $\alpha$, the aggregate volume fraction as determined by the fitting process, increased with shear rate, although aggregates remained smaller (Fig. 6B).

Direct imaging showed that once a suspension had reached a steady-state viscosity, the vast majority of microrods were in aggregates (Fig. 4). Mass conservation and the calculated aggregate density, along with the assumption of complete aggregation, allow an estimation of the overall suspension volume fraction that is filled with aggregates. A KriegerDougherty-type constitutive relationship that is commonly used to describe the impact of suspended particles on the viscosity of a suspension ${ }^{21}$ was combined with the calculated aggregate
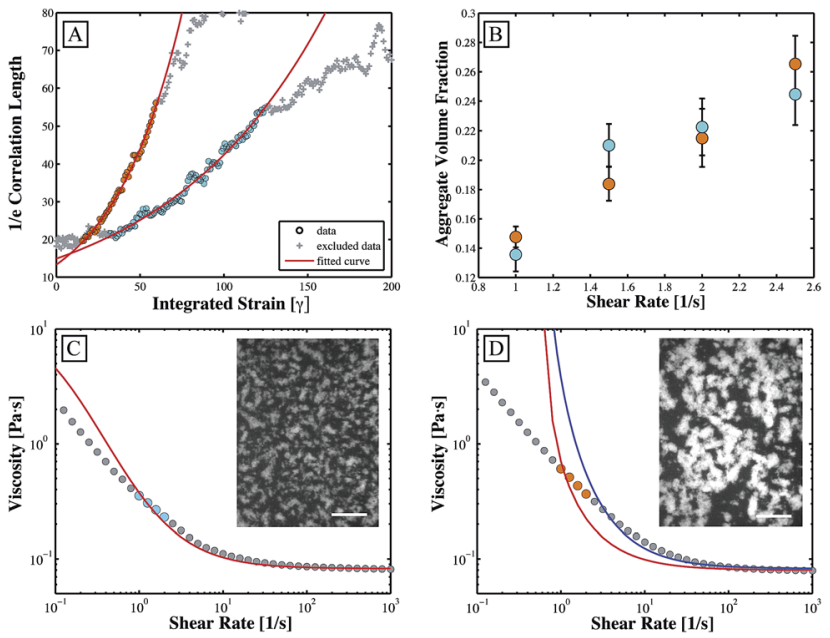

Fig. 6 Early stage aggregation curves (A) closely follow the aggregation model (eqn (4)) in the scaling region. Representative data are shown for $\phi=0.07$ (orange) and $\phi=0.04$ (turquoise) at $\dot{\gamma}=1 \mathrm{~s}^{-1}$. The fits reveal an aggregate density that increases with shear rate (B). Krieger-Dougherty (KD) derived fits that take into account increasing aggregate density with shear rate (eqn (5)) work well for lower concentrations $(\mathrm{C})$ where the model does not predict a divergent viscosity at a positive shear rate. High concentrations (D) deviate significantly from the predicted behavior since eqn (5) diverges at a positive shear rate. This is consistent with the system evolving into a gel-like state ( $D$, inset) which would not be described by eqn (5). Scale bars represent $1 \mathrm{~mm}$.

density to yield a shear rate dependent viscosity enhancement with one free parameter, the intrinsic viscosity, $[\eta]$.

$$
\frac{\eta}{\eta_{0}}=\left(1-\frac{\phi}{\phi_{\max }}\right)^{-[\eta] \phi_{\max }}=\left(1-\frac{\phi_{\mathrm{rods}}}{\phi_{\mathrm{agg}}(\dot{\gamma}) \phi_{\max }}\right)^{-[\eta] \phi_{\max }}
$$

In this equation, $\phi_{\max }$, the maximal packing fraction of the system, at which point the viscosity diverges, was set equal to 0.62 in order to be near the value for random close packing of spheres. $[\eta]$, the intrinsic viscosity, is a geometric factor that measures the solute's contribution to the overall suspension viscosity. ${ }^{20} \phi_{\text {rods }}$ and $\phi_{\text {agg }}$ are the volume fraction taken up by the rods themselves and the shear-rate dependent steady-state aggregate volume fraction, respectively, determined from the calculated aggregate density as described above.

Eqn (5) fits the steady-state shear thinning curve for low concentrations quite well, with an intrinsic viscosity of 4.23 , but breaks down for larger ones (Fig. 6C and D), because of the divergence at a shear rate near the fit region. Therefore, to construct a model curve for the high concentration sample, the intrinsic viscosity determined from the low concentration fit was used instead (shown as the blue curve in Fig. 6D). This method yields a better representation of the underlying physics and agrees better with the measured rheology than the one built from the high concentration's fit parameters, suggesting that the value of 4.23 is a reasonable overall estimate of the intrinsic viscosity of SU-8 microrod aggregates formed under shear. This is in contrast to the reported result of $[\eta] \approx 20$ for carbon nanotube suspensions obtained by appealing to a dilute-limit 


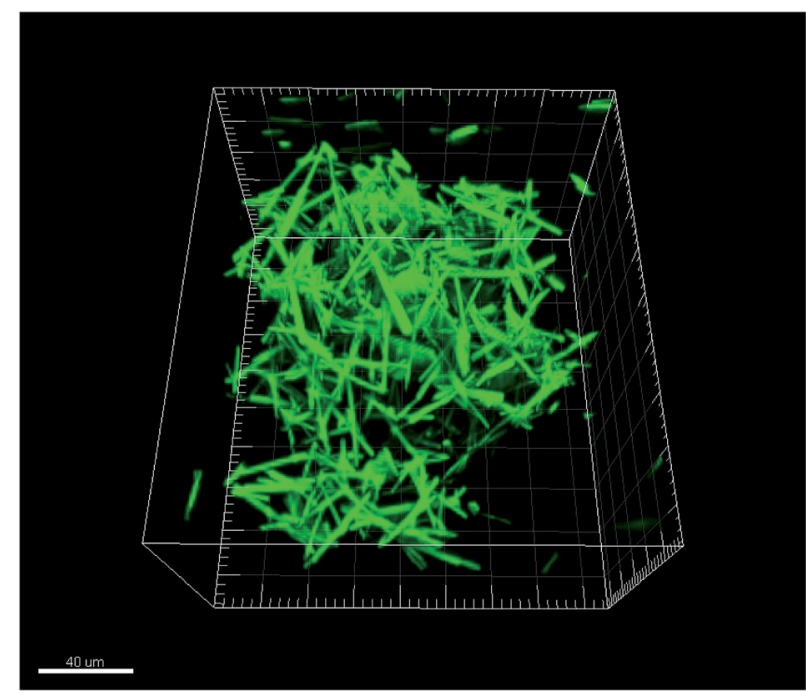

Fig. 7 The 3D surface structure of an SU- 8 microrod aggregate formed at a shear rate of $0.5 \mathrm{~s}^{-1}$ and captured using a confocal microscope and appears highly disordered. In movies, structures such as this one appear to dramatically disrupt the surrounding flow field. $\dagger$

relationship, ${ }^{22}$ suggesting that details of material properties and deformation mechanics may generate substantial intrinsic viscosity differences between aggregates that are, at first glance, geometrically similar.

Under high magnification using confocal microscopy, the aggregates appeared to be disordered, porous structures with limited surface ordering (Fig. 7). Close examination of the dynamics of the regions around the aggregates via confocal rheology showed dramatic fluctuations in the flow field (see ESI movie $\dagger$ ), consistent with the above model's approximation that no fluid flows through the porous aggregates, and therefore their presence increases the volume fraction of the suspension. A direct reliable measurement of aggregate density via confocal microscopy is not possible due to the refractive index mismatch between the rods and the surrounding fluid, but images of the outer shell like the one depicted in Fig. 7 are at least roughly consistent with the aggregate density predicted by the model.

\section{Discussion}

The combination of an aggregate density that grows with shear rate and the assumption of complete aggregation at steady-state leads to a viscosity model that is consistent with experimental data over a wide range of shear rates. While perhaps counterintuitive, the positive correlation between the shear rate and the aggregate density is consistent with previous analyses of fractal dimensions in aggregates of spherical particles. ${ }^{8}$ For high aspect ratio particles, the increase in aggregate density may be the result of rearrangements in the aggregate structure immediately following the collision process. An initial encounter between a microrod and an aggregate will result in a single contact, but subsequent motion due to fluid stresses would allow for an increase in the number of contacts, which can be quite high for assemblies of high aspect ratio particles. The number of contacts necessary to fully stabilize the particles will be higher at higher shear rates, producing denser aggregates.

At very low shear rates and high concentrations, where the Krieger-Dougherty-based model appears to break down, aggregates are sufficiently large that the system may be in a more gel like state. This is supported both qualitatively by the structure of the high concentration system at low shear rates (Fig. 6D, inset), as well as theoretically in that the model at high concentration predicts a divergence of the viscosity at a positive shear rate. In a hard sphere or similar structurally static suspension, this discontinuity would represent a space filling and therefore arrested system. Measured viscosity in this context would be a consequence of gel deformation rather than drag on suspended particles.

While the simple model presented is encouraging, especially since it allows for the estimation of the intrinsic viscosity, $[\eta]$, there are some potential weaknesses inherent in the assumptions. For example, relaxing the assumption that every encountered rod is added to an aggregate (by introducing a sticking probability) results in a functional form identical to eqn (4) except with an additional collision probability in the exponent, which would produce similar fits but lower values for the aggregate density. While in reality the sticking probability is undoubtedly a function of shear rate and aggregate size, the success of the model presented here suggests that it may not be a major effect for this system.

\section{Conclusion}

The combination of rheological measurements and quantitative image analysis allows for direct study of the structural dynamics present during shear-induced aggregation, as well as their association with bulk material properties. The micron-scale, non-Brownian suspension of SU-8 microrods utilized here provides a useful balance between the faithful modeling of industrially relevant materials and accessibility to confocal and wide-field microscopy that enables experimental examination of these microscopic dynamics in greater detail than has previously been available.

We have presented direct measurements of the growth in aggregate size as a function of integrated strain, and shown that a simple porous-growth model is sufficient to describe early stage aggregation within our system, allowing us to conclude that the aggregate density increases with shear rate. Using this result we have presented an augmented Krieger-Dougherty model that describes the shear-thinning behavior of aggregating rod suspensions reasonably well at concentrations and shear rates for which the aggregates behave as independent particles in suspension rather than an interconnected gel.

These results constitute a quantitative, direct experimental demonstration of the association between aggregation dynamics and viscosity enhancement in a rod suspension, and demonstrate how the combination of quantitative imaging and rheology can help advance the fundamental understanding of how the dynamic structural features of complex fluids influence their material properties. 


\section{Acknowledgements}

This work was supported by the AFOSR (FA9550-10-1-0473). JSU is supported in part by the Georgetown Interdisciplinary Chair in Science Fund. We thank David Gribbin for assistance with microrod fabrication.

\section{References}

1 M. J. Solomon and P. T. Spicer, Soft Matter, 2010, 6, 13911400.

2 J. Mewis and N. J. Wagner, J. Non-Newtonian Fluid Mech., 2009, 157, 147-150.

3 S. Goto, H. Nagazono and H. Kato, Rheol. Acta, 1986, 25, 246256.

4 S. Majumdar, R. Krishnaswamy and A. Sood, Proc. Natl. Acad. Sci. U. S. A., 2011, 108, 8996.

5 G. Kwon, Y. Heo, K. Shin and B. J. Sung, Phys. Rev. E: Stat., Nonlinear, Soft Matter Phys., 2012, 85, 011143.

6 E. K. Hobbie, Rheol. Acta, 2010, 49, 323-334.

7 K. M. Yearsley, M. R. Mackley, F. Chinesta and A. Leygue, J. Rheol., 2012, 56, 1465-1490.

8 P. Bubakova, M. Pivokonsky and P. Filip, Powder Technol., 2013, 235, 540-549.

9 V. A. Tolpekin, M. H. G. Duits, D. van den Ende and J. Mellema, Langmuir, 2004, 20, 2614-2627.

10 J. C. Flesch, P. T. Spicer and S. E. Pratsinis, AIChE J., 1999, 45, 1114-1124.
11 C. F. Schmid, L. H. Switzer and D. J. Klingenberg, J. Rheol., 2000, 44, 781.

12 C. F. Schmid and D. J. Klingenberg, Phys. Rev. Lett., 2000, 84, 290-293.

13 R. G. Alargova, V. N. Paunov and O. D. Velev, Langmuir, 2006, 22, 765-774.

14 S. K. Dutta, A. Mbi, R. C. Arevalo and D. L. Blair, Rev. Sci. Instrum., 2013, 84, 063702.

15 R. de Rooij, A. A. Potanin, D. van den Ende and J. Mellema, J. Chem. Phys., 1993, 99, 9213.

16 M. Bercea and P. Navard, Macromolecules, 2000, 33, 60116016.

17 S. S. Rahatekar, K. K. K. Koziol, S. A. Butler, J. A. Elliott, M. S. P. Shaffer, M. R. Mackley and A. H. Windle, J. Rheol., 2006, 50, 599.

18 M. Yamanoi, C. Leer, F. W. J. van Hattum, O. S. Carneiro and J. M. Maia, J. Colloid Interface Sci., 2010, 347, 183-191.

19 A. Karppinen, T. Saarinen, J. Salmela, A. Laukkanen, M. Nuopponen and J. Seppälä, Cellulose, 2012, 19, 18071819.

20 D. B. Genovese, Adv. Colloid Interface Sci., 2012, 171-172, 1-16.

21 A. Puisto, X. Illa, M. Mohtaschemi and M. J. Alava, Eur. Phys. J. E: Soft Matter Biol. Phys., 2012, 35, 6.

22 S. Lin-Gibson, J. Pathak, E. Grulke, H. Wang and E. Hobbie, Phys. Rev. Lett., 2004, 92, 048302. 